\title{
Torus Figure Generating System Based on Graph Theoretical Representation and Topological Analysis
}

\author{
Kento TARUI *1 • Fangyan DONG *1 • Yutaka HATAKEYAMA *2 • \\ Kaoru HIROTA *1
}

\begin{abstract}
A trick art drawing system based on a torus figure generating algorithm is constructed. The proposed algorithm is based on the shapes and positions of corners of torus figures. The proposed system is able to generate an arbitrary $\mathrm{n}$-bar torus type possible or impossible figure using interactive mouse-clicking operations on a figure generated from $\mathrm{n}+1$ initial inputs only. It is possible to draw a trick art figure by painting each face of the obtained graph. The proposed system can serve as basic tool for experiments on visual psychology and an educational support tool for discrete mathematics classes.
\end{abstract}

Keywords : graph theory, knot theory, impossible figures, computer vision, visual psychology

\section{Introduction}

An impossible figure is a line drawing that seems to be a polyhedron, but actually represents an object which cannot exist in 3 dimensional reality. Impossible figures are one of the most distinctive examples of illusionism. Most studies in this area are based on a visual psychology perspective $[15,16]$. Using the shape from line drawing method, commonly used in 3D drawing approaches, leads to erroneous results when attempted with impossible figures [9-12]. Addressing this problem is a significant research issue with applications in 3D modeling and pattern recognition in computer vision. More specifically, [6-8] show the algebraic and combinatorial approaches used to determine whether a line drawing, all of whose vertices are consistent with the dictionary of trihedral vertices $[5,7]$, is an impossible figure or not. In either case, the main line of investigation in this research is to decide whether a given line drawing is an impossible figure or not and why it is impossible. However, the capacity to distinguish possible and impossible figures does mean that it is possible to au-

*1 Department of Computational Intelligence and Systems Science, Interdisciplinary Graduate School of Science and Engineering, Tokyo Institute of Technology

*2 Center of Medical Information Science, Medical School, Kochi University tomatically generate such a line drawing, since set of randomly located lines that make no sense for a human-being also violates the conditions for being a possible (real world) 3D figure. The condition that the figure has to look like a polyhedron is important for generating such an impossible figure.

This paper first examines the requirements for a figure to look like a polyhedron as is consistent with the dictionary of trihedral vertices for every vertex in the figure. Then a generating system is proposed that produces torus figures obtained by connecting the ends of some quadratic prisms with the uniform rectangular cross-sections in a toroidal form. The resulting figures are either possible figures called possible torus figures, or correspond to impossible figures obtained by twisting and crossing possible ones, and are called impossible torus figures. The latter are the main types of impossible figures that are investigated.

A torus figure can be determined uniquely by the shape and the coordinates of every corner and the information of whether a bar is the upper or lower one at every crossing of bars which isn't a corner. Among the required three conditions, the shape of every corner is generally difficult to express. A braids theory based representation of the shape of corners is proposed in $[2,3]$ and a method to represent the shapes of corners with regular triangular tiles is introduced 
in [14] by restricting corners to having only three kinds of angles. In [13], a method is proposed to represent a corner with arbitrary angles based on graph theory that requires less information than the braids based approach because information about edges' crossings is not used in a graph theoretical representation. This paper proposes an algorithm to generate the corresponding torus figures from the required three conditions, where the shapes of corners are represented by the graph theoretical method introduced in [13].

A torus figure generating system based on the proposed algorithm is implemented on a $\mathrm{PC}$ using $\mathrm{C}++$ with Open GL and the Qt GUI library. The generating system requires two kinds of inputs, the number of bars and coordinate of every corner, e.g. $N+1$ inputs are required to generate an $\mathrm{N}$-bar torus figure. From these two types of input, the system automatically calculates a possible combination of simple junctions that can be used to construct corners and determine the upper and lower bar of every crossing and then generates a torus figure. Every corner's shape, as well as the upper and lower bars of a crossing in the obtained figure can be changed by mouse clicking operations, so any user can generate an arbitrary torus figure.

Because every generated torus figure from the proposed system is only a line drawing, a function to put colors and textures to the generated figure is implemented as an additional feature of the generating system.

Investigations of representation and topological properties of torus figures are stated in 2 . The proposed algorithm and specification of a torus figures' automatic generating system are introduced in 3 . An additional function concerning coloring and textures of the proposed system and several example figures are shown in 4 .

\section{Graph Theoretical Representa- tion and Topological Analysis}

\subsection{Torus figures}

A possible torus figure is defined as a projection (parallel projection, general position, see [6]) of a polyhedron connecting quadrangular prisms that have uniform rectangular cross sections in a toroidal form (Fig.1) .

An impossible figure that looks like a twisted or crossed possible torus figure is called impossible torus

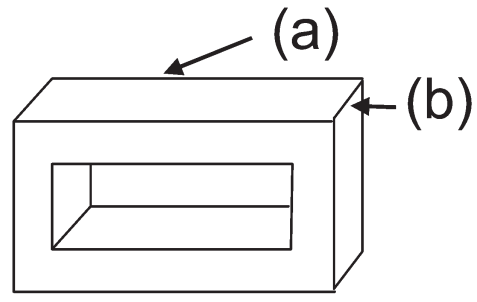

Fig.1 Example of a possible torus figure

(a) Longitudinal edge, (b) Transversal edge

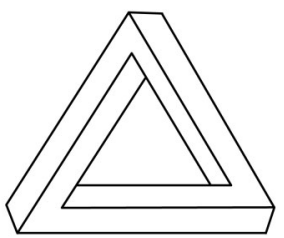

(a)

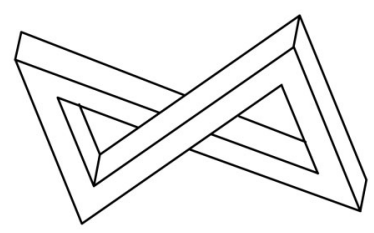

(b)
Fig.2 Impossible torus figures resulting from (a) twisting and (b) crossing

figure. A possible torus figure that has been twisted and/or crossed is said to be an impossible torus figure (Fig.2).

Both possible and impossible torus figures are referred to as torus figures.

The definition above is too intuitive from the mathematical point of view. It is necessary to construct a generating system to determine the scope of the definition of torus figures. However, there is no well-defined and consensus-based definition of torus figures. Therefore the following two hypotheses concerning to multibar figures are formulated to constrain the scope of target figures in accordance with [13].

(Hypothesis 1) Every longitudinal edge including hidden edges is a projection of a generating line of quadrangular prisms that have the uniform rectangular cross sections and has connections each other at a corner.

(Hypothesis 2) Every vertex is compatible with the vertex dictionary [5] [7] of trihedral vertices.

Hypothesis 2 is a constraint for a figure to look like a polyhedron and to exclude irrelevant figures such as Fig.3 (a). Hypothesis 1 is a constraint to make the figure to look like a multibar figure and to exclude figures that are obtained by connecting quadrangular prisms but do not look like multibar figures such as Fig.3(b). 


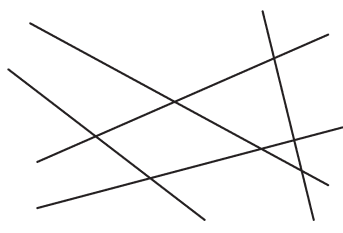

(a)

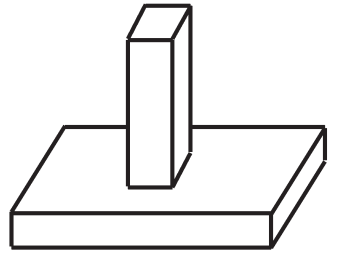

(b)
Fig.3 Example of excluded figures

\subsection{Torus type graphs and thier link structure}

As an abstract representation of the connection information of longitudinal edges in torus figures, torus type graphs are defined in accordance with [13], based on bars and simple junctions that are graphs corresponding to longitudinal edges of quadrangular prisms and their connections.

A bar is defined as a bipartite graph with four mutually separated edges whose partite sets have four vertices each one. Therefore, these four edges induce a bijection between the two partite sets. These two partite sets are called bases. A simple junction is also a bipartite graph with mutually separated four edges that consists of two bases, with each one from a different bar. Based on the concept of bars and simple

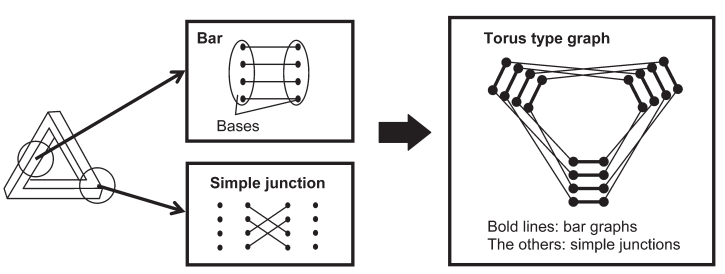

Fig.4 An example of Definition 1-3

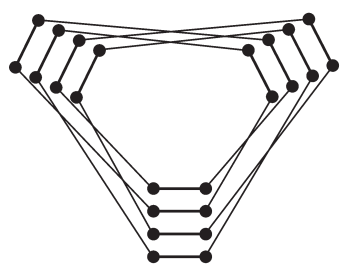

(a)

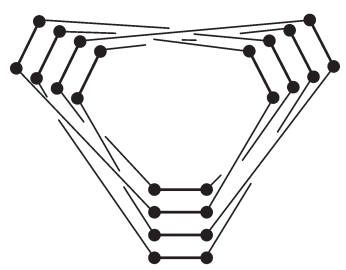

(b)
Fig.5 An example of 3-bar torus type graph. (Bold lines : bar graphs, normal lines: simple junctions) (a) 3-bar torus type graph and (b) its link structure junctions, a torus type graph is defined as a graph consisting of $\mathrm{n}$ bars and $\mathrm{n}$ simple junctions that are connected to form a loop (see Fig.4).

Every torus graph is uniquely determined by the order of its simple junctions up to the order of bars and edges of each bar.

Because a spatial embedding of every torus type graph is a link, it is called a link structure of the torus type graph (see Fig.5). Every torus figure can be classified by the number of components of any link structure of the corresponding torus type graph, since the number of components is one of the most typical invariants of links.

Considering torus figures, it is known from [13] that the number of components of their link structures is restricted to 1,2 or 4 .

\subsection{Shape of corners}

There exist 24 possible simple junctions. However, not every simple junction has a corresponding corner in torus figures. Simple junctions that construct corners are shown in Fig.6. Two bars that are connected by the junction are drawn as a set of four parallel lines. Then, those lines are connected in accordance with the junction and as a consequence, four vertices are generated at the connected points of the lines. Finally, two transversal edges are drawn between the four vertices. There are six patterns to draw these edges and if one of them makes every four vertices consistent with the dictionary of trihedral vertices, the junction is determined to be a junction that constructs a torus figure corner.

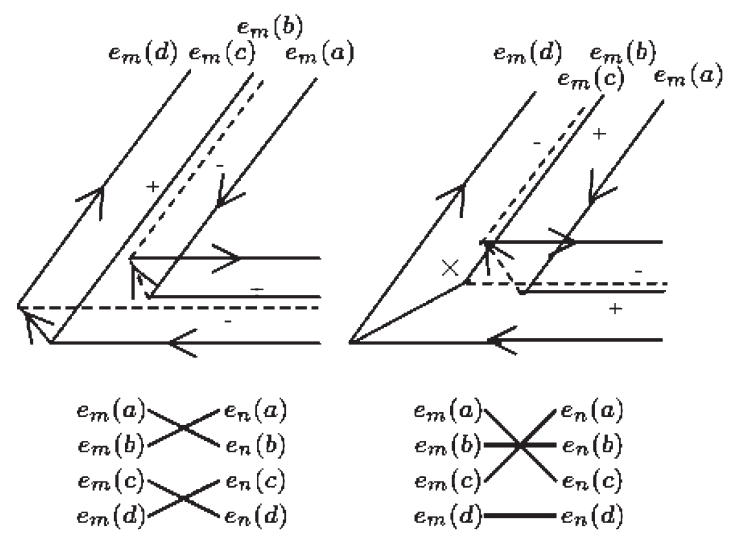

Fig.6 Simple junctions satisfying the conditions. (Left) Labelable, (Right) Unlabelable 

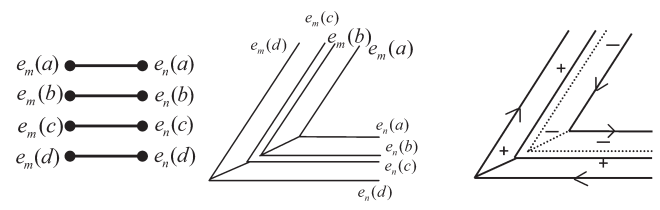

(i) Type 1
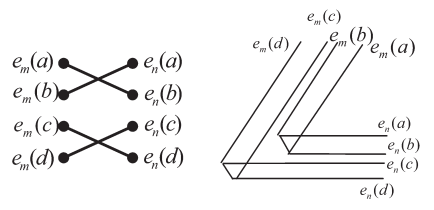

(ii) Type 2
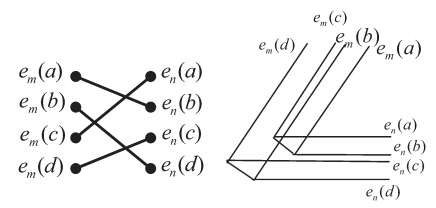

(iii) Type 3
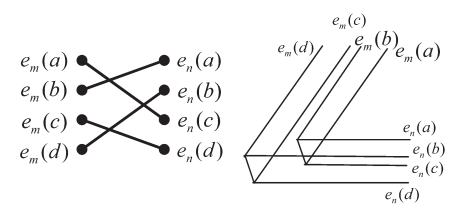

(iv) Type 4
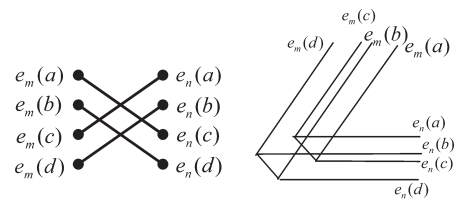

(v) Type 5
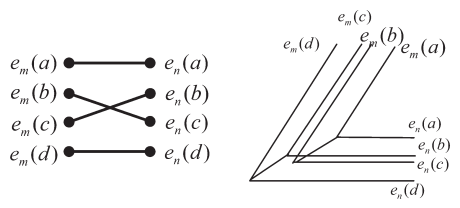

(vi) Type 6
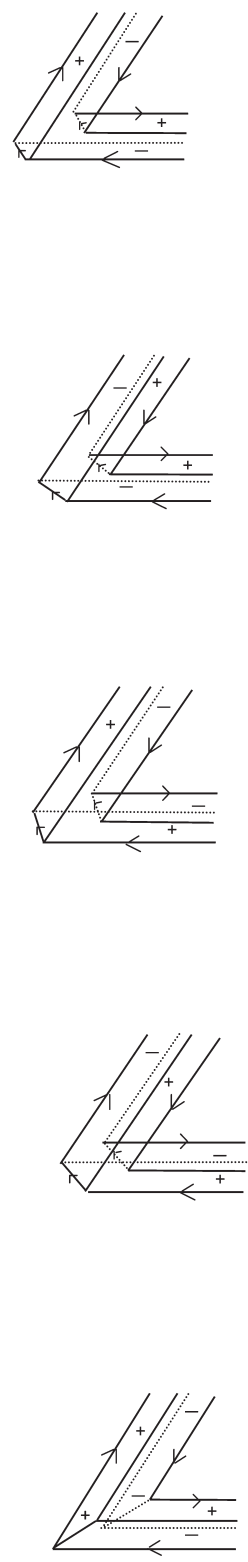

(i)

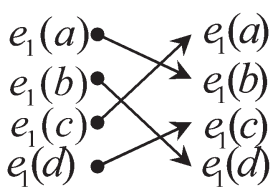

(iii)

Theorem 1 below:

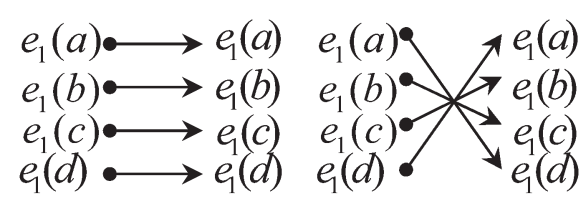

It is shown in [13] that the 6 simple junctions which can construct corners of torus figures can be classified into 6 categories as shown in (Fig.7). Edge $e_{m}$ (b) and $e_{m}(c)$ can be chosen as a hidden edge to make a labelable figure. For Type 1 and Type 6 corners, there are two ways to draw transversal edges, but for the other corner type there is only one.

A torus type graph that has only Type 1-6 simple junctions is said to be a normal form torus type graph. The term torus type graph refers to the normal form torus type graph hereinafter.

\subsection{Properties of torus figures}

In this section, relations between combinations of simple junctions and torus figures are analyzed and several theorems that are necessary to construct a torus figure generating system are proved.

In a torus graph, every edge on the first bar is contained in a cycle constructed with the following bars and simple junctions. Theorem 1 shows a relation between torus figures and such patterns of edge connections.

A torus type graph has corresponding torus figures iff the torus type graph satisfies the conditions

(1) The connection pattern of edges represented by its simple junctions belongs to the list shown in Fig. 8,

(2) It contains an even number of Type 2 , Type 5 , and Type 6 simple junctions.

Before proceeding to the proof, the following lemma [13] needs to be introduced.

(ii)

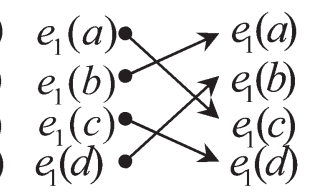

(iv)

Fig.8 Four connection patterns of simple junctions that represent torus figures
Fig.7 List of shapes of corners. (Left) simple junctions (Middle) examples of corresponding corners (Right) labeled figures in the case of hidden $e_{m}(b)$ 


\section{Lemma1}

A torus figure can be embedded in a toroidal surface.

If a torus figure is embedded in a toroidal surface in accordance with the algorithm introduced in the proof of this lemma, visible edges appear on the upper half surface and hidden edges appear on the lower one of the toroidal surface (Fig.9).

Proof of the Theorem 1

(Necessity) (1) There are eight possible connection patterns in Fig.10 for arbitrary combinations of Type 1-6 torus type graphs [1].

Let's suppose there is an $N$-bar $(N>3)$ torus figure whose corners correspond to the simple junction set $\mathbf{J G}_{T}$. The torus figure is embeddable to a toroidal surface as stated in the Lemma. Then the corresponding torus type graph $G_{T}\left(J_{G_{T}}\right)$ should also be embeddable to the surface.

If the connection pattern of $\mathbf{J G}_{T}$ is the case shown in Fig.10 (vi), $e_{1}(a)$ returns to $e_{1}(b)$ and $e_{1}(b)$ returns to $e_{1}(a)$. Then, tracing the belt region between $e_{1}(a)$ and $e_{1}(b)$, the region constructs a nonorientable surface on the toroidal surface. This leads to a contradiction because the toroidal surface is orientable. Therefore, the connection pattern of $\mathbf{J G}_{T}$ is not the case shown in Fig.10(vi). In the same way, the connection pattern of $\mathbf{J} \mathbf{G}_{T}$ is neither (v), (vii), nor (viii) . Finally, the connection patterns of longitudinal edges of torus figures are shown in Fig.8.

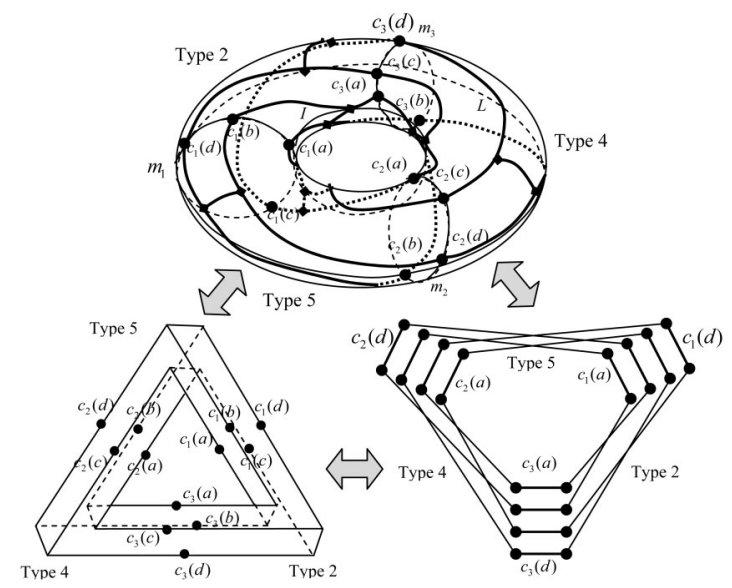

Fig.9 Example of torus figure embedded in a toroidal surface

(Left) Torus figure, (Center) Embedding of the torus figure, (Right) Corresponding torus type graph

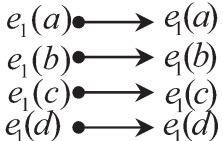

(i)

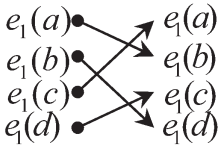

(iv)

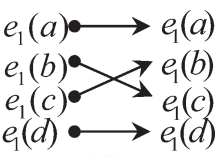

(vii)

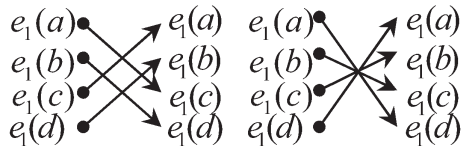

(ii)

(iii)

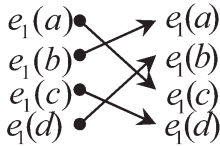

(v)

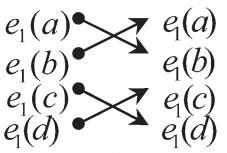

(vi)
Fig.10 Possible connection patterns of simple junctions

(2) When Type 2, Type 5 and Type 6 simple junctions are embedded in a toroidal surface, if $e_{1}(b)$ is a visible edge then $e_{2}(c)$ is a visible edge, and vice versa (see Fig.9). On the other hand, for Type 1, Type 3, Type 4 simple junctions, if $e_{1}(b)$ is a visible edge then $e_{2}(b)$ is a visible edge. In the case that the torus graph contains an odd number of Type 2, Type 5, and Type 6 simple junctions, if $e_{1}(b)$ is a visible edge then $e_{1}$ (b) becomes a hidden edge. This is a contradiction. Therefore, the number of Type 2, Type 5, and Type 6 simple junctions is even.

(Sufficiency) Let $G_{T}\left(\mathbf{J G}_{T}\right)$ be a $N$-bar $(N>3)$ torus type graph satisfying (1) and (2).

Four lines connected as shown in Fig. 8 can be embedded in a toroidal surface. Hence, condition (1) assures that there exists an embedding of $G_{T}\left(\mathbf{J G}_{T}\right)$ in a toroidal surface. Therefore, the existence of corresponding torus figures is shown by investigating embeddings in a toroidal surface. At first, $G_{T}\left(\mathbf{J G}_{T}\right)$ is embedded in a toroidal surface with the following procedure.

It can be shown that every Type of simple junctions can be embedded in the toroidal surface as (d) edges corresponding to visible edges are embedded on upper surface, hidden edges are embedded on lower surface when a corner is constructed from a simple junction according to the method described in 2.3. Meanwhile, lines corresponding to edges that become visible from hidden and hidden from visible are embedded as across $L$ or $I$. If every $c_{k}(x)$ is considered as the contraction of an edge of $B_{k}$, Fig. 8 can be considered as a proof for Type 2, 4 and 5 simple junctions 
and a proof for the other simple junctions can be given in the same way. Because the positions of $c_{1}(x)$ and $c_{2}(x)$ are decided by $J G_{1}$, the following junction is embedded depending on $c_{2}(x)$ and determines $c_{3}(x)$. Condition (2) assures that the positions of $c_{n}(x)$ and $c_{1}(x)$ do not lead to a contradiction with the embedding of $J G_{n}$. It implies that a torus figure can be obtained by connecting the constructed corners from $\mathbf{J G}_{T}$ according to the method described in 2.3. Therefore, a torus type graph satisfying (1) and (2) has corresponding torus figures. (Q.E.D.)

There are various combinations of simple junctions that are consistent with the connection patterns in Fig.8. It takes long time to calculate them. Therefore, an approach to replace a junction graph to another that keeps consistency of all of them with the patterns in Fig. 8 is required. Theorem 2 shows the restrictions of such a replacement of simple junctions.

Theorem 2

(i) There are two groups of mutually replaceable simple junctions, (a) Type 1, 3, and 4 and (b) Type 2, 5 , and 6 .

(ii) If an element in (a) is replaced by one in (b), two junctions in each group should be replaced at once.

Proof

(i) First, every simple junction can not be replaced with another one of the other group because the number of Type 2, 5, and 6 simple junctions is changed to an odd number. On the other hand, they are mutually replaceable in their own group without any change of the number of Type 2, 5, and 6 junctions. For every connection pattern in Fig.8, let's suppose that the simple junctions are ordered with the replaced junction is at the end. It is clear that the connection pattern of the new simple junctions set is also one of the patterns shown in Fig.8 by replacing the junction and investigating the connection pattern (Fig.11).

(ii) This replacement does not change the number of Type 2, 5, and 6 simple junctions. It is also shown that the connection pattern of the new simple junctions set is also included in Fig. 8 by applying the procedure described in (i) twice. (Q.E.D.)

The basis for generating combinations that represent torus figures is given by the discussions above. Therefore, if the coordinates of all junctions and information of crossing of all bars are controlled in the

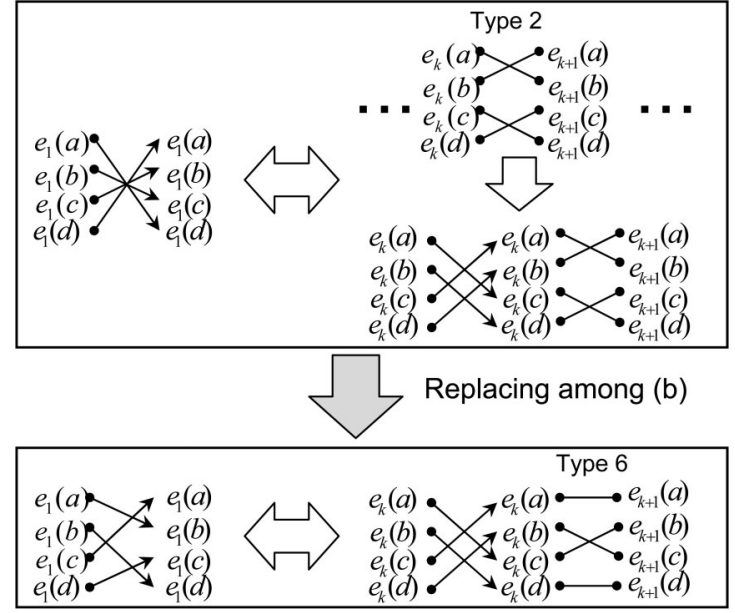

Fig.11 Connections patterns by replacing a junction

system, the system is able to generate arbitrary torus figures.

\subsection{Conditions for the number of faces}

If the user wants to set colors or texture images on the faces of torus figures, it is necessary to extract face information from generated figures. In this section, Theorem 3 about conditions for the number of faces of generated torus figures is demonstrated. Because this theorem requires detailed information about transversal edges, it is necessary to distinguish the way to put transversal edges to Type 1 and Type 6 simple junctions (Fig.12). Concerning to the other simple junctions, the way is uniquely determined.

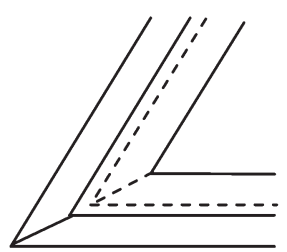

Type 1

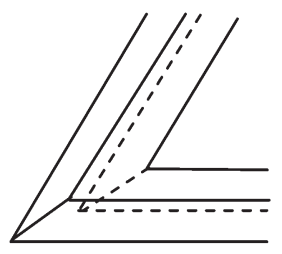

Type 6

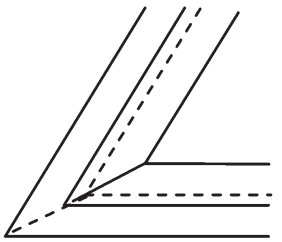

Type 1'

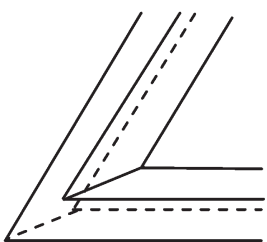

Type 6'
Fig.12 Transversal edges of Type 1 and Type 6 


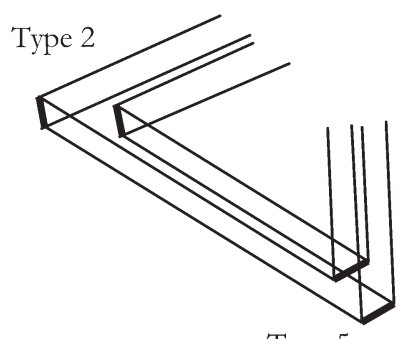

(i)

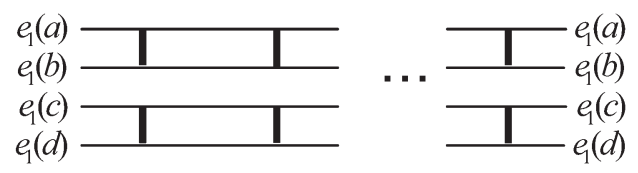

(iii)

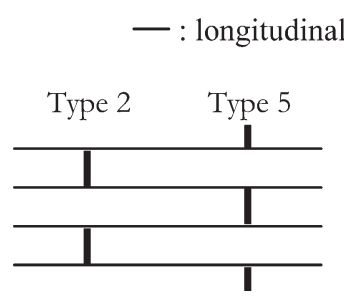

(ii)

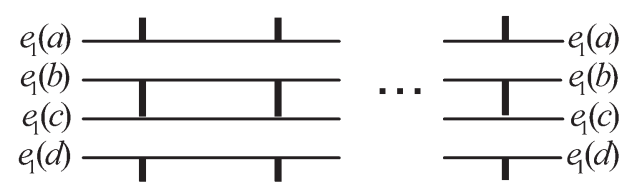

(iv)

Fig.13 Number of faces and transversal edges

(i), (ii) : Example of (c), (iii), (iv) : Without (a) - (h)

\section{Theorem 3}

If a link structure of the corresponding torus type graph of a torus figure with $N$-bars has 1 , 2, or 4 components and the relations listed from (a) to (h) below are satisfied in its simple junctions, the number of faces of the figure is 2 . Otherwise it equals $2 N+2$.

(a) The $i$ th simple junction is Type 1 and the $i+$ 1th simple junction is Type 1', $3,5,6$ '

(b) The $i$ th simple junction is Type 1' and the $i+$ 1th simple junction is Type $1,2,4,6$

(c) The $i$ th simple junction is Type 2 and the $i+$ 1th simple junction is Type 1', $3,5,6$ '

(d) The $i$ th simple junction is Type 3 and the $i+$ 1th simple junction is Type 1', 3, 5, 6'

(e) The $i$ th simple junction is Type 4 and the $i+$ 1th simple junction is Type $1,2,4,6$

(f) The $i$ th simple junction is Type 5 and the $i+$ 1th simple junction is Type $1,2,4,6$

(g) The $i$ th simple junction is Type 6 and the $i+$ 1th simple junction is Type $1,2,4,6$

(h) The $i$ th simple junction is Type 6' and the $i+$ 1th simple junction is Type $1^{\prime}, 3,5,6$ '

Proof

Because every torus figure is embeddable on a toroidal surface and the genus of arbitrary torus figure is one, the Euler number of the figure should equal zero or two. When it is zero, the number of the faces is $2 N$, otherwise it is $(2 N+2)$.

It is shown in [13] that the number of faces of the figure is $2 N$ in the case of 1 or 2 components. The proof in the case of 4 components is as follows:

Because of the Lemma, a torus figure is embeddable in a toroidal surface. Therefore, it is necessary to demonstrate whether every surface of the embedded torus figure is 2-fold or not to determine its Euler number.

In the case of 4 components, if the torus figure is embedded in a toroidal surface as in the same way as described in the Lemma, four belts bounded $2 N$ longitudinal edges respectively are obtained by ignoring the transversal edges. If every belt has transversal edges, every region of the embedded toroidal surface is 2 -fold and then the Euler number is zero. By mathematical induction, if there is no adjacent simple junctions from (a) to (h), there exists a belt that includes no transversal edges, otherwise one edge exists on every belt at least (Fig.13). (Q.E.D.)

\section{Torus Figures Generating System}

The constraints of torus type graphs that have corresponding torus figures are shown in section 2. By distinguishing Type 1 simple junctions from Type 1' and Type 6 from Type 6 in a torus type graph that has corresponding torus figures as shown in Fig.12, the shape of each corner of the corresponding torus figures is determined by the torus type graph since each transversal edges is unique for each simple junction. Therefore, crossings of bars and coordinates of 
corners are obtained and the corresponding unique torus figures are determined. In this section, a system to generate $N$ bar torus figures from a torus type graph information of crossings and coordinates is proposed.

\subsection{Torus figures generating algorithm}

The proposed system implements a modified version of the algorithm introduced in [13] for generating torus figures. The improved algorithm receives $\mathrm{N}$ +1 inputs and generates a $N$ bar torus, as follows :

Conditions :

- Number of bars: $N$ (Note: $B_{i}$ shows the $i$-th bar with edges $\left[E_{i}(a), E_{i}(b), E_{i}(c), E_{i}(c)\right]$.)

-Simple junction set : $\mathbf{J}=\left[J_{1} \cdots J_{N}\right]$, where $J_{i} \in$ \{Type 1, 1', 2, 3, 4, 5, 6, 6'\} (Note $: J_{i}: B_{i} \rightarrow$ $B_{i+1}$ and $J_{i}^{-1}: B_{i} \rightarrow B_{i-1}\left(B_{N+1}=B_{1}, B_{1-1}=B_{N}\right)$.)

- Coordinates of corners : $\mathbf{B P}=\left[B P_{1} \cdots B P_{N}\right]$, where $B P_{i}=\left(x_{i}, y_{i}\right)$ (Note: Every adjacent three points are not on the same line.)

-Table of crossings : $\mathrm{C}=\left[C_{1} \cdots C_{M}\right]$, where $C_{m}=(d$ $\left.\left(B_{i}\right), d\left(B_{j}\right)\right)$ is a crossing of $B_{i}$ under $B_{j}$

-Thickness parameters : $\alpha, \beta(\beta>\alpha \geq 0)$

- Hidden edges : calculated considering $E_{1}(b)$ as a hidden edge.

\section{Algorithm ::}

$/{ }^{*}$ Calculation*/

(1) Calculate the lines $E_{1}=\overline{B P_{1} B P_{2}}, E_{2}=\overline{B P_{2} B P_{3}}$, - . , $E_{N}=\overline{B P_{N} B P_{1}}$.

(2) Calculate the lines $\mathbf{E}_{i}=\left\{E_{i}(x) \mid x=a, b, c, d\right\} / /$ $E_{i}$ with $d\left(E_{i}(a), E_{i}\right)=d\left(E_{i}(d), E_{i}\right)=\beta, d\left(E_{i}(b), E_{i}\right)=$ $d\left(E_{i}(c), E_{i}\right)=\alpha, d\left(E_{i}(a), E_{i}(d)\right)=2 \beta$, and $d\left(E_{i}(b)\right.$, $\left.E_{i}(c)\right)=2 \alpha$ for $1 \leq i \leq N$, under the condition that if the lengths of lines are adjusted to connect $E_{i}(a)$, $E_{i+1}(a)$ and $E_{i}(d), E_{i+1}(d)$, these four edges do not cross except at these two points.

(3) Calculate the junction points $J P_{i}\left(E_{i}(x)\right)$ as the crossing point of $E_{i}(x)$ and $J_{i}\left[E_{i}(x)\right] \in \mathbf{E}_{i}$ for $x=a$, $b, c, d$ and $1 \leq i \leq N$.

(4) Recalculate $E_{i}(x)=\overline{J P_{i-1}\left(J_{i-1}^{-1}\left[E_{i}(x)\right] J P_{i}\left(E_{i}(x)\right)\right.}$ as a longitudinal edge for $x=a, b, c, d$ and $1 \leq i \leq N$ $\left(J P_{N+1}=J P_{1}, J_{N+1}^{-1}=J_{1}^{-1}\right)$.

(5) Calculate the transversal edges $T E_{i}^{1}=\overline{J P_{i}\left(E_{i}(a)\right)}$ $\overline{J P_{i}\left(E_{i}(b)\right)}, T E_{i}^{2}=\overline{J P_{i}\left(E_{i}(c)\right) J P_{i}\left(E_{i}(d)\right)}$ if $J_{i}=$ Type 1 , 2 , 4, or 6, else $T E_{i}^{1}=\overline{J P_{i}\left(E_{i}(a)\right) J P_{i}\left(E_{i}(c)\right)}, T E_{i}^{2}=$ $\overline{J P_{i}\left(E_{i}(b)\right) J P_{i}\left(E_{i}(d)\right)}$, for $1 \leq i \leq N$.

(6) Calculate the crossings $\mathbf{C}_{1} \cdots \mathbf{C}_{N}$ s.t. $\mathbf{C}_{i}$ is the as- cending ordered set of $C_{m}=\left(d\left(B_{i}\right), d\left(B_{j}\right)\right)(\in \mathrm{C})$ with $d\left(B_{i}\right)$, for $1 \leq i \leq N$.

(7) Calculate the sub-longitudinal edges $S E_{i}(x)=$ $S E_{i}^{1}(x) \cup \cdots \cup S E_{i}^{\mid M_{i}+1}(x)\left(M_{i}=\left|\mathbf{C}_{i}\right|\right)$ s.t. $S E_{i}^{1}(x)=\overline{J P_{i-1}}$ $\overline{\left(J_{i-1}^{-1}\left[E_{i}(x)\right] C P_{j 1}\left(E_{i}(x)\right)\right.}$ for $m=1, S E_{i}^{m}(x)=\overline{C P_{j m-1}^{\prime}}$ $\overline{\left(E_{i}(x)\right) C P_{j m}\left(E_{i}(x)\right)}$ for $1<m<M_{i}$, and $S E_{i}^{M_{i}}(x)=$ $\overline{C P_{j m}^{\prime}\left(E_{i}(x)\right) J P_{i}\left[E_{i}(x)\right]}$ for $m=M_{i}$, where $C P_{j m}\left(E_{i}(x)\right)$ and $C P_{j m}^{\prime}\left(E_{i}(x)\right)$ are the crossing points of $E_{i}(x)$ and $E_{j m}(a)$ (denoted as $\left.C P\left(E_{i}(x), E_{j m}(a)\right)\right)$ and $C P\left(E_{i}(x)\right.$, $\left.E_{j m}(d)\right)$, respectively, at $C_{i m}=\left(d\left(B_{i}\right), d\left(B_{j m}\right)\right)$ $\left(\in \mathrm{C}_{i}\right)$, if $d\left(J P_{i-1}\left(J_{i-1}^{-1}\left[E_{i}(x)\right]\right), C P\left(E_{i}(x), E_{j m}(a)\right)\right)$ $<d\left(J P_{i-1}\left(J_{i-1}^{-1}\left[E_{i}(x)\right]\right), C P\left(E_{i}(x), E_{j m}(d)\right)\right)$, else $C P$ $\left(E_{i}(x), E_{j m}(d)\right)$ and $C P\left(E_{i}(x), E_{j m}(a)\right)$, respectively. /*Drawing*/

(8) For $1 \leq i \leq N$, if $d\left(E_{i}(a), B P_{i+1}\right)<d\left(E_{i}(d), B P_{i+1}\right)$ $\left(B P_{N+1}=B P_{1}\right)$,

if $J_{i}=$ Type 1 or 6 and $E_{i}(b)$ is hidden, draw transversal edge $T E_{i}^{1}$, else draw $T E_{i}^{2}$, and if $d$ $\left(E_{i}(a), B P_{i+1}\right)>d\left(E_{i}(d), B P_{i+1}\right)\left(B P_{N+1}=B P_{1}\right)$,

if $J_{i}=$ Type 1 or 6 and $E_{i}(c)$ is hidden, draw transversal edge $T E_{i}^{2}$, else draw $T E_{i}^{1}$.

(9) For $1 \leq i \leq N$,

draw sub-longitudinal edges $S E_{i}(x)$ except, $\overline{J P_{i-1}\left(J_{i-1}^{-1}\left[E_{i}(x)\right]\right) J P_{i}\left(E_{i}(x)\right)^{\prime}}$, if $J_{i-1}^{-1}\left[E_{i}(x)\right]$ and $E_{i}(x)$ have an intersection $J P_{i}\left(E_{i}(x)\right)^{\prime} \neq J P_{i-1}\left(J_{i-1}^{-1}\left[E_{i}(x)\right]\right)$ and $J_{i-1}^{-1}\left[E_{i}(x)\right]$ is a hidden transversal edge or except $\overline{J P_{i}\left(E_{i}(x)\right) " J P_{i}\left(E_{i}(x)\right)}$ if $J_{i}\left[E_{i}(x)\right]$ and $E_{i}(x)$ have an intersection $J P_{i}\left(E_{i}(x)\right) " \neq J P_{i}\left(E_{i}(x)\right)$ and $J_{i}\left[E_{i}(x)\right]$ is a hidden transversal edge for $x \in\{a, d\}$.

draw $S_{i}(x)$ if $E_{i}(x)$ is not a hidden edge, for $x$ $\in\{b, c\}$.

(END)

When $e_{1}(c)$ is a hidden edge, the corresponding torus figure can be drawn in the same way. Fig.14 shows a simple overview of applying this algorithm.

In what follows, an implementation of proposed algorithm is discussed. 


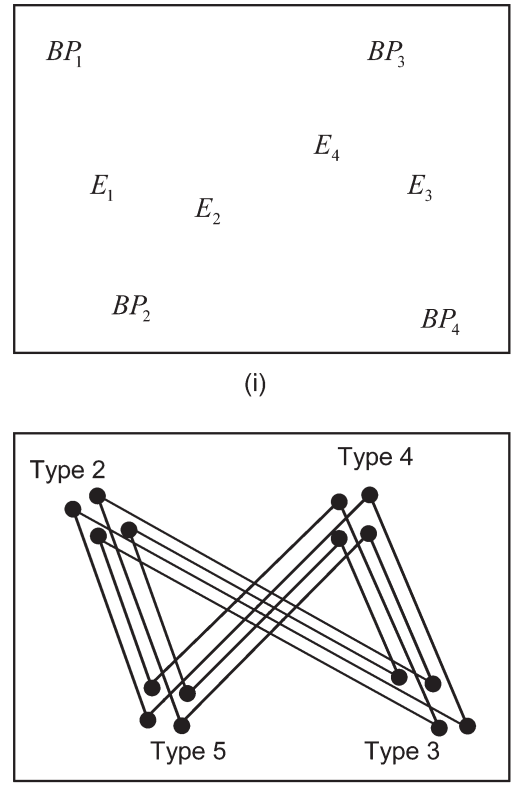

(iii)

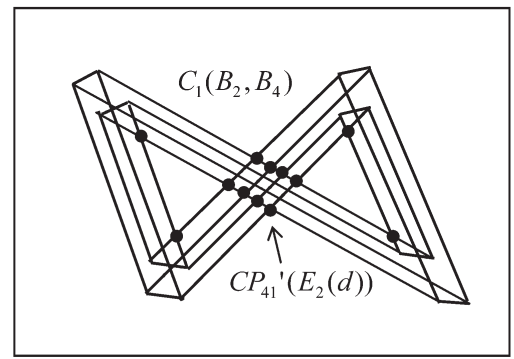

(v)

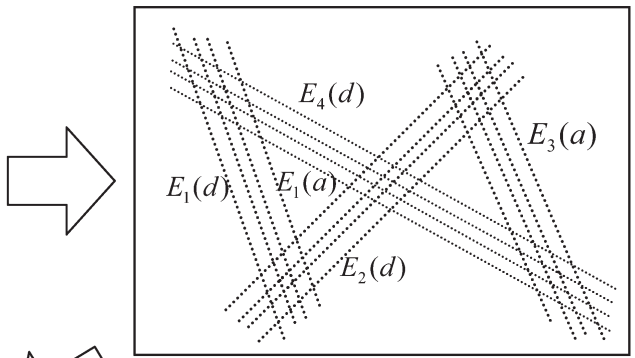

(ii)

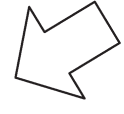

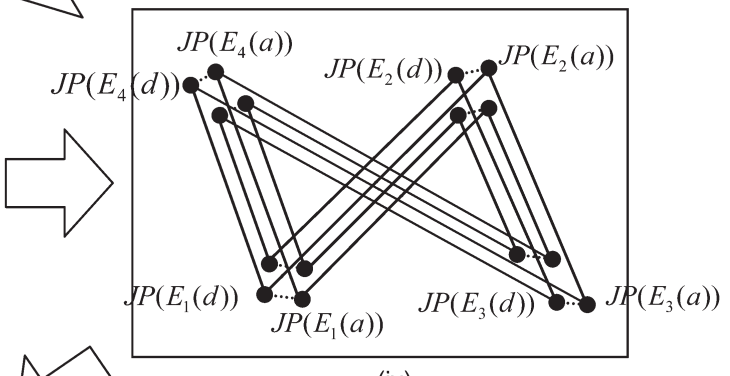

(iv)

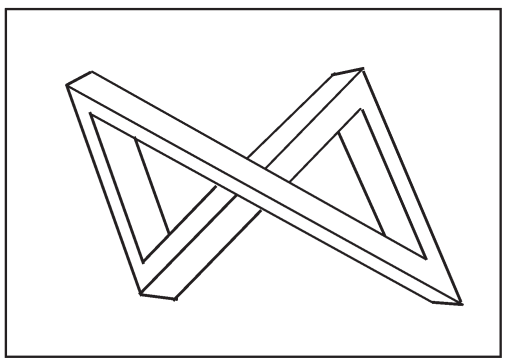

(vi)

Fig.14 Overview of the algorithm
(Calculation) : (i) (1)
(ii) (2)
(iii) (3), (4)
(iv)
(5), (v) (6),
(7), (Drawing) : (vi)
$(8),(9)$

\subsection{User interface of the torus figures generating system}

If a list of simple junctions, a list of coordinates of corners, and a table of crossings are input, the corresponding torus figure is generated using the algorithm proposed in 3.1. The input information, however, requires the user to select a list of simple junctions from $8^{N}$ candidates to generate an $N$-bar torus figure and, as shown in Theorem 1 , not all candidates have corresponding torus figures. Furthermore, the crossings of bars depend on the complete coordinates of the corners, except the above and the below of bars at each crossing.

Therefore, the proposed system requires only two kinds of input: the number of bars $N$, and the $N$ coordinates of the corners. Once the number of bars is given, a simple junction set that satisfies Theorem 1 can be calculated by searching the $6^{N}$ space, since whether a simple junction set has corresponding torus figures does not depends on the transversal edges. When the coordinates of the corners are obtained, which pair of bars has crossing can be determined. The system calculates a simple junction set and a table of crossings from the inputs and then outputs a torus figure automatically. In case the output is not satisfactory, the user can change the undesired parts of corners and crossings. The replacement candidates are calculated in accordance with Theorem 2. A conceptual diagram of the system is shown in Fig.15.

The proposed system provides an interactive graphic user interface (GUI) to set information that determines a torus figure. An example of the process 


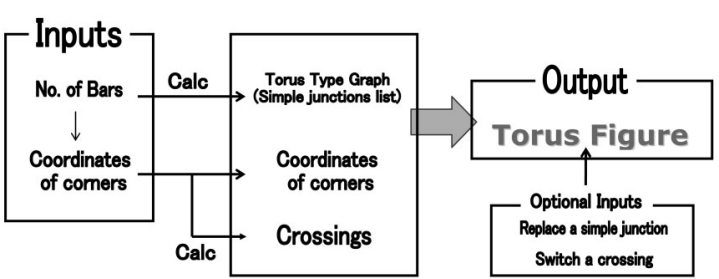

Fig.15 Conceptual diagram of the proposed torus figures generating system

of generating a torus figure is shown below in screenshots.

Step $1:$ Initial Input

The user inputs the number of bars (Fig. 16). The choice is determined after clicking the OK button after selecting from the options using the arrows to the right. Numbers from 3 to 15 can be selected, the lower bound is theoretical minimum and the upper bound is arbitrary due to visibility limitations of generated figures.

Step $2:$ Input of the position of the corners

The user inputs the coordinates of each corner connecting two bars in the drawing window (Fig. 17).

The polygon is closed when the designated number of vertices (Step 1) is input. (Fig. 18).

To draw the torus figure, the user must press the keyboard shortcut Ctrl+D or select the option "Draw" from the menu bar. The system selects the first simple junction that satisfies Theorem 1 from the possible combinations and then calculates a combination of simple junctions and crossings to output a default torus figure (Fig.19). Three figures are displayed in the output window. The one in the upper left of the display is a torus figure showing the hidden edges. The other two figures, at the lower part of the display, are torus figures obtained by eliminating the hidden edges from the upper figure. This can be done by choosing either $e_{1}(b)$ or $e_{1}(c)$ as a hidden edge. The remaining hidden edges are determined automatically since they depend on this choice, as mentioned in 3.1. The two lower figures can be considered as the two sides of a coin.

The described steps result in an acceptable torus figure. If the user is not satisfied with the output figure, changes in crossings and shapes of corners can be implemented as follows.

Step 3 : Switching at crossing

If the user wants to swap the top and bottom bars at a crossing, a simple click on it will do it (Fig.20).

Step 4 : Replacing junctions

If a change in the shape of a corner is desired, the user should right-click on such corner to view a list of shapes which can replace it, in accordance with Theorem 2 (Fig.21(a)). Items under the separator in the menu do not comply with Theorem 2 (ii), and, if the user decides to change the corner into one of such Type, it is necessary to replace two junctions. In this case the system displays a dialog window for the selection of the junction to be replaced with the chosen Type of simple junction (Fig.21(b)).

Therefore, an arbitrary torus figure is obtained. Operations of Step 3 and 4 are in no particular order.

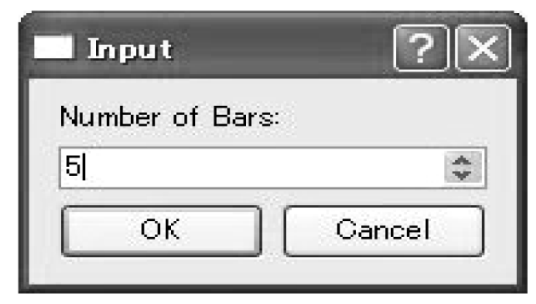

Fig.16 Input window of the number of bars

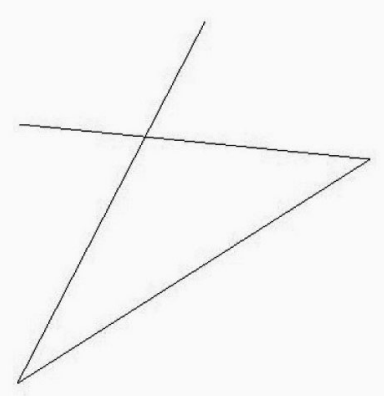

Fig.17 Input of the position of corners

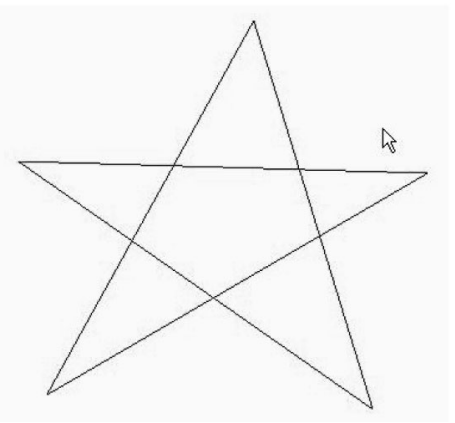

Fig.18 Topology of a torus figure 

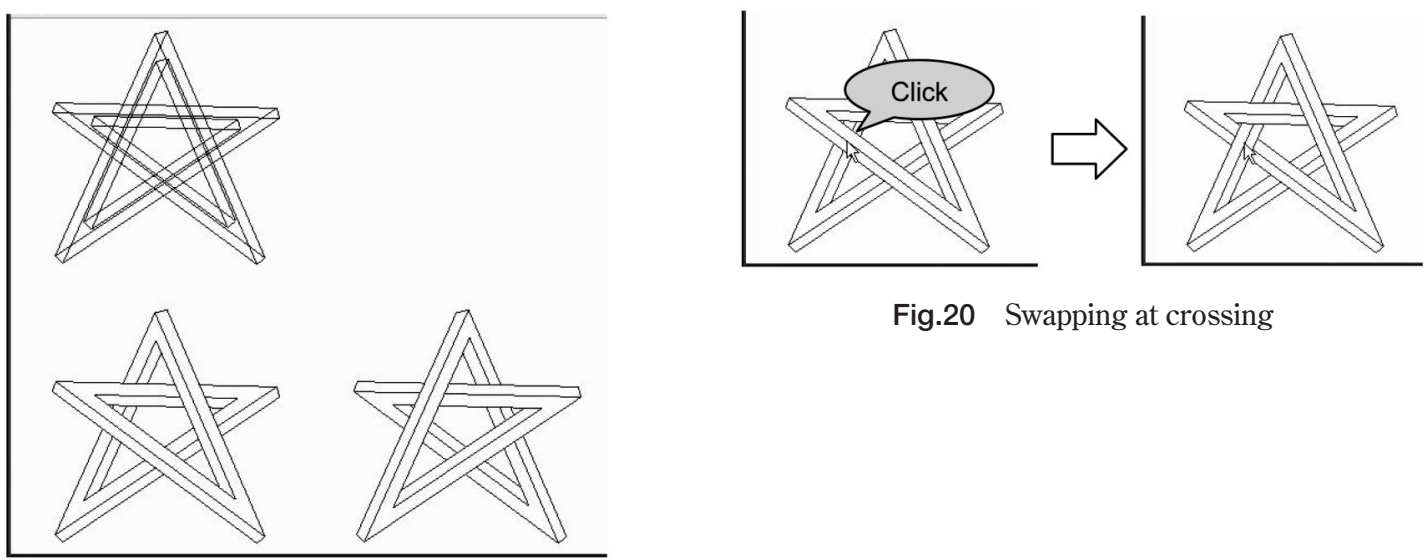

Fig.20 Swapping at crossing

Fig.19 Output.

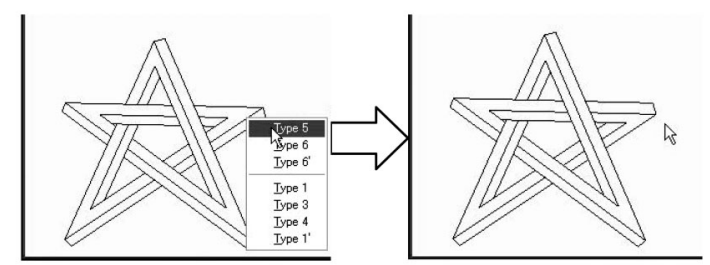

(a)
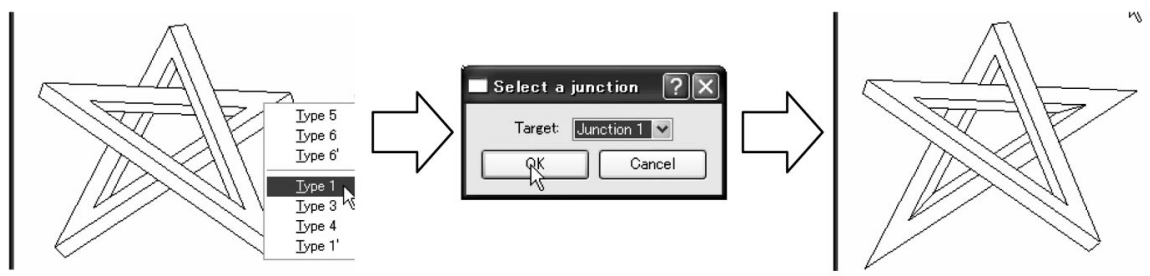

(b)

Fig.21 Replacing a simple junction with one of the (a) same group (b) different groups

\section{Examples of generated figures}

\subsection{Coloring and texture}

Torus figures generated by the system that is introduced in 3 are only line drawings. Therefore, for the purpose that the generated figures become to look like trick arts, additional functions to put colors and textures to them are shown below.

First of all, the function to decorate each face of the generated figures is introduced. According to Theorem 3 , the number of the faces of a generated figure is obtained. This face decorating function is implemented by assigning information of color and texture to the list with which length is the obtained number. The user interface is shown in Fig.22.
When a user does double-click on a desiring face to decorate, the dialog of asking which is desired to use color or texture to decorate the selected face is appeared. After the user selects one of them, the dialog to choose desiring color from the color list or texture image file from the user's directory is appeared. After the user finishes the selection, the corresponding decorated figure is obtained.

Proposed system also has a function to decorate background (Fig.23). The user interface is the same as the case of faces except that user should do doubleclick on the background area. 


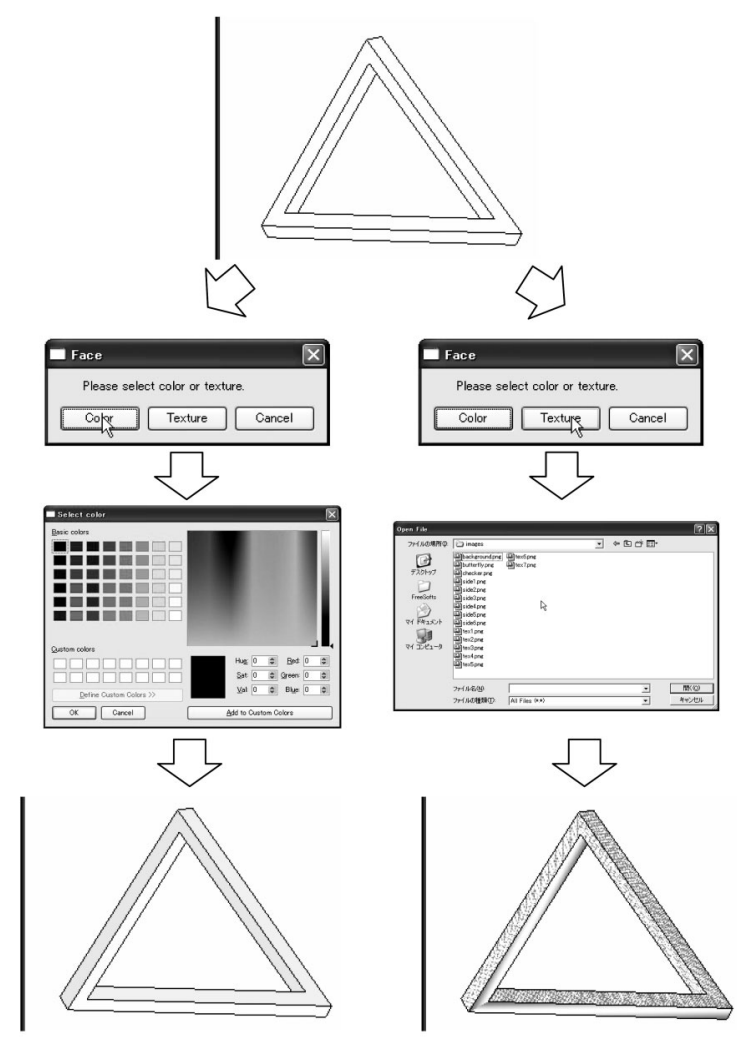

Fig.22 Face decorating function

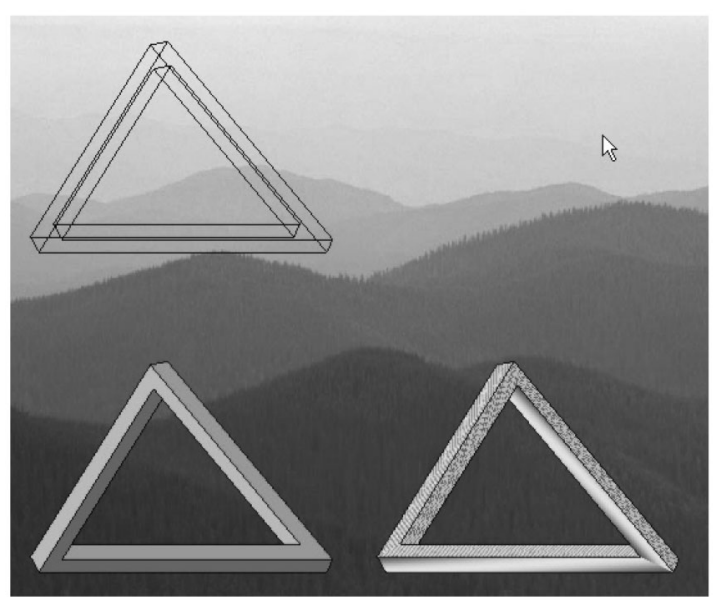

Fig.23 Decoration of the background

\subsection{Drawing decorated figures}

Examples that have visual effect by decorating obtained torus figures are shown below.

Figure 24(a) shows the structure of the Escher's water fall. By decorating the faces of the figure, the structure becomes more obvious (Fig.24(b)).

Other examples are shown in Fig.25. Figure 25(a)

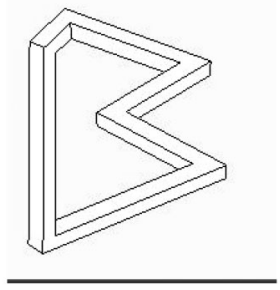

(a)

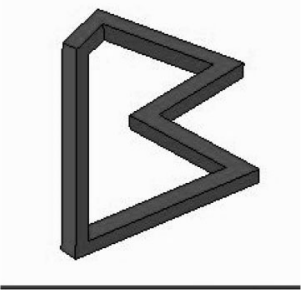

(b)
Fig.24 Structure of the Escher's waterfall

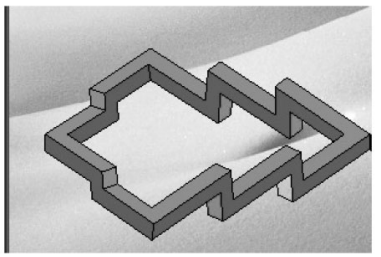

(a)

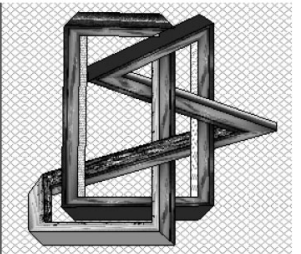

(b)
Fig.25 Examples of torus figure with decoration

shows the structure of Escher's staircases and Fig.25 (b) and (c) shows a torus figure constructing a knot. A torus figure generating system based on the proposed algorithm is implemented on $\mathrm{PC}$ by $\mathrm{C}++$ programming language with Open GL graphic library and Qt GUI library. The output is obtained within a second after user gives input in every step of using the system.

\section{Conclusion}

A system that automatically generates torus figures, one of the main focal points in the study of impossible figures, is implemented on a PC using the $\mathrm{C}++$ language with the Open GL graphic library and the $\mathrm{Qt}$ GUI library. Given a number of bars and coordinates for every corner, the system constructs a torus figure by calculating a possible combination of simple junctions that can form corners according to Theorem 1 , as well as the upper and lower bars for crossings which aren't corners. Every corner's shape can be changed by clicking the right button of the mouse around the corner and selecting changeable simple junctions calculated according to Theorem 2 . The upper and lower of bars of every crossing can be changed by clicking the left button around the crossing. An arbitrary torus figure can thus be generated by using the proposed 
torus figures generating system whose functioning is ensured theoretically by Theorem 1 and Theorem 2 .

The system also makes it possible to put colors and textures on each face of a generated torus figure by double clicking the desirable face and selecting the desirable color or texture. Because every edge of a torus figure is represented as the edge of a graph, it is possible to detect an arbitrary face as a region of a graph and decorate its interior. Theorem 3 is used to detect all faces in the generated figure and to keep the information of each face in the system. The performance of this function is evaluated by generating figures (shown in section 4) that look like Escher's waterfall and stair cases, which are two of the most well known impossible figures.

Any operation in the proposed system takes less than a second to generate the output once the user inputs data.

The proposed method can contribute to various activities in the visual entertainment industry, because it makes it possible to evaluate the attractiveness of a generated figure by adding colors and textures to it. Furthermore, the proposed system can be useful as a basic tool for experiments on visual psychology and as support for computer based educational tools for discrete mathematics. Indeed, any arbitrary torus figure that one wishes to investigate is obtained in realtime. It is also possible to calculate how many of the torus figures generated from all the possible combinations of simple junctions correspond to impossible figures when the number of bars is fixed, regardless of any conditions that might be imposed on the coordinates of the corners and the upper and lower bars of crossings.

The proposed algorithm can be extended for graphs containing 3-junctions by expanding the vertex dictionary from just trihedral to multi-hedral one.

\section{References}

[1 C.C.Adams, The Knot Book, pp.1-30, Freeman, 2001.

[2] T.M. Cowan, The theory of braids and the analysis of impossible figures, Journal of Mathematical Psychology, Vol. 11, pp.190-212, 1974.

[ 3 ] T.M. Cowan, Organizing the properties of impossible figures, Perception, Vol.6, pp.41-56, 1977.
[ 4 ] B. Mohar and C. Thomassen, Graphs on Surfaces, pp.3-98, Johns Hopkins University Press, 2001.

[ 5 ] D.A. Huffman, Impossible objects as nonsense sentences, Machine Intelligence, Vol. 6, pp.295-323, 1971.

[6] K. Sugihara, "Three-dimensional realization of anomalous pictures-An application of picture interpretation theory to toy design”, Pattern Recognition, Vol. 30, No.7, pp.1061-1067, 1997.

[ 7 ] K. Sugihara, Studies on mathematical structures of line drawings of polyhedra and their applications to scene analysis (Japanese), Researches of the Electrotechnical Laboratory, No.800, pp.1-22, 1979.

[ 8 ] K. Sugihara, Classification of impossible objects, Perception, Vol. 11, pp.65-74, 1982.

[ 9 ] L. Ros and F. Thomas, Overcoming superstrictness in line drawing interpretation, IEEE Trans. Pattern Analysis and Machine Intelligence, vol. 24, pp.456466, 2002.

[10] J. Liu and Y.T. Lee, A graph-based method for face identification from a single 2D line drawing, IEEE Trans. Pattern Analysis and Machine Intelligence, vol. 23, pp. 1106-1119, 2001.

[11] J. Liu and X.T. Tang, Evolutionary search for faces from line drawings, IEEE Trans. Pattern Analysis and Machine Intelligence, vol. 27, pp. 861- 872, 2005.

[12] Y. Sun and Y.T. Lee, Topological analysis of a single line drawing for 3D shape recovery, Proc. GRAPHITE, pp.167-172, 2004.

[13] K. Tarui et al., Graph/knot theoretical analysis and generation for impossible figures, Journal of Advanced Computational Intelligence and Intelligent Informatics, Vol.11, No.10, 2007.

[14] D. Uribe, A set of impossible tiles, Proceeding of the third international conference mathematics \& design 2001.

[15] Z. Kulpa, Putting order in the impossible, Perception, vol. 16, pp. 201-214, 1987.

[16] L. S. Penrose and R. Penrose, Impossible objects - A special type of visual illusion, British Journal of Psychology, Vol. 49, pp.31-33, 1958.

(2008年 1 月 6 日 受付)

(2008年 3 月12日 採録)

[Contact Address]

G3-49, 4259 Nagatsuta, Midori-ku, Yokohama-city 226-8502, Japan

Department of Computational Intelligence and Systems Science, Interdisciplinary Graduate School of Science and Engineering, Tokyo Institute of Technology

Kento TARUI

TEL : 045-924-5682

FAX : 045-924-5676

E-mail : taru@hrt.dis.titech.ac.jp 


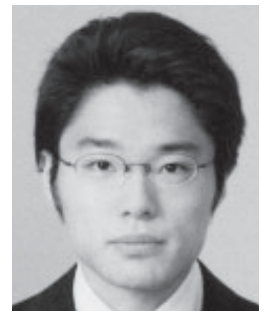

\section{Kento TARUI [member]}

received the B.S. degree from Tokyo University of Science and M.E. from Tokyo Institute of Technology, Japan, in 2003 and 2004, respectively. He has been a Ph.D. course student at Tokyo Institute of Technology, Japan since 2005. His reseach interests are computer vision and computational geometry.

\section{Yutaka HATAKEYAMA [member]}

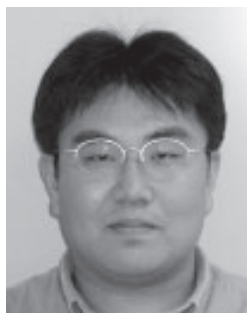

received the B.E.,M.E., and Dr. Eng. degrees from Tokyo Institute of Technology, Japan, in 1999, 2001, 2004 , respectively. He is currently an associate professor with Kochi university. His research interests are image processing and medical informatics.

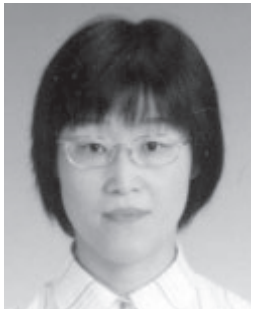

\section{Fangyan DONG [member]}

was born in China. She received the masters degree from Beihang University (June,1991), Dr. degree from Tokyo Institute of Technology in 2003. From 2003 to Apr., 2006, she did post-fellow research at Tokyo Tech. Since May, 2006, she is working at the department of computational intelligence and systems science, Tokyo Inst. of Tech. as an assistant professor. Her research interests include computational intelligence, logistics optimization, and intelligent robot. She is a member of Japan Society for Fuzzy Theory and Intelligent Informatics, Japanese Society for Artificial Intelligence, and Information Processing Society of Japan.

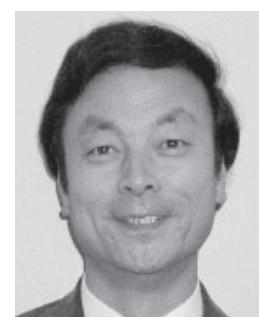

\section{Kaoru HIROTA [member]}

received Dr. E. degree from Tokyo Institute of Technology in 1979. After his career at Sagami Institute of Technology and Hosei University, he has been with Tokyo Institute of Technology. Currently he is a professor at the department of Computational Intelligence and Systems Science, Interdisciplinary Graduate School of Science and Engineering, Tokyo Institute of Technology. His research interests include fuzzy systems, intelligent robot, and image understanding. He experienced president-elect and fellow of IFSA (International Fuzzy Systems Association), and president of SOFT (Japan Society for Fuzzy Theory and Systems.) He is a chief editor of $\mathrm{J}$. of Advanced Computational Intelligence and Intelligent Informatics. Banki Donat Medal, Henri Coanda Medal, Grigore MOISIL Award, SOFT best paper award, Acoustical Society of Japan best paper award, honorary professorships from de La Salle University and Changchun Univ. of Science \& Technology, and Honoris Causa from Bulacan state university were awarded to him. He organized more than 10 international conferences/symposiums as a founding/general/program chair. He has been publishing about 200 journal papers, 50 books, and 450 conference papers. 